\title{
A correlation between the heavy element content of transiting extrasolar planets and the metallicity of their parent stars
}

\author{
T. Guillot ${ }^{1}$, N. C. Santos ${ }^{2,3,4}$, F. Pont ${ }^{3}$, N. $\operatorname{Iro}^{5}$, C. Melo $^{6}$, and I. Ribas ${ }^{7}$ \\ 1 Observatoire de la Côte d'Azur, CNRS UMR 6202, 06304 Nice Cedex 4, France \\ e-mail: guillot@obs-nice.fr \\ 2 Centro de Astronomia e Astrofísica da Universidade de Lisboa, Observatório Astronómico de Lisboa, Tapada da Ajuda, \\ 1349-018 Lisboa, Portugal \\ 3 Observatoire de Genève, 51 Ch. des Maillettes, 1290 Sauverny, Switzerland \\ 4 Centro de Geofisica de Evora, Rua Romeo Ramalho 59, 7002-554 Evora, Portugal \\ 5 Department of Physics, University of Florida, PO Box 118440, Gainesville 32611-8440, USA \\ 6 European Southern Observatory, Casilla 19001, Santiago 19, Chile \\ 7 Institut d'Estudis Espacials de Catalunya - CSIC, Campus UAB, Facultat de Ciències, Torre C5-parell-2a, 08193 Bellaterra, Spain
}

Received 22 April 2006 / Accepted 30 April 2006

\section{ABSTRACT}

\begin{abstract}
Context. Nine extrasolar planets with masses between 110 and $430 M_{\oplus}$ are known to transit their star. The knowledge of their masses and radii allows an estimate of their composition, but uncertainties on equations of state, opacities and possible missing energy sources imply that only inaccurate constraints can be derived when considering each planet separately.

Aims. We seek to better understand the composition of transiting extrasolar planets by considering them as an ensemble, and by comparing the obtained planetary properties to that of the parent stars.

Methods. We use evolution models and constraints on the stellar ages to derive the mass of heavy elements present in the planets. Possible additional energy sources like tidal dissipation due to an inclined orbit or to downward kinetic energy transport are considered. Results. We show that the nine transiting planets discovered so far belong to a quite homogeneous ensemble that is characterized by a mass of heavy elements that is a relatively steep function of the stellar metallicity, from less than 20 earth masses of heavy elements around solar composition stars, to up to $\sim 100 M_{\oplus}$ for three times the solar metallicity (the precise values being model-dependant). The correlation is still to be ascertained however. Statistical tests imply a worst-case $1 / 3$ probability of a false positive.

Conclusions. Together with the observed lack of giant planets in close orbits around metal-poor stars, these results appear to imply that heavy elements play a key role in the formation of close-in giant planets. The large masses of heavy elements inferred for planets orbiting metal rich stars was not anticipated by planet formation models and shows the need for alternative theories including migration and subsequent collection of planetesimals.
\end{abstract}

Key words. planets and satellites: formation - stars: abundances - planetary systems

\section{Introduction}

Transiting extrasolar planets are extremely interesting objects to study because the coupling of radial velocity and photometric measurements allows a determination of both their masses and radii, and thus, in principle, a constraint on their composition. For that purpose, we need to know as accurately as possible the properties of the star-planet systems, and to apply evolution models using up-to-date input physics.

Nine transiting extrasolar planets are known to date. In Table 1, we summarize their characteristics and that of their parent stars on the basis of available literature, including recently derived metallicities and stellar effective temperatures from high-resolution and high signal-to-noise spectra obtained with UVES for the faint stars OGLE-TR-10, 56, 111, 113 and TrES-1 (Santos et al. 2006) and OGLE-TR-132 (Pont et al. 2006). The ages were derived from isochrone fitting when possible. In some cases, lower limits were obtained from lithium abundances, the Ca II activity-age relation, and an analysis of the stellar rotational velocity (Melo et al. 2006). In the absence of useful constraints, we used a conservative upper limit of $10 \mathrm{Ga}$ because the stars' metallicities and proper motions imply that they all belong to the galactic thin disk.

The radii of close-in extrasolar giant planets (hereafter Pegasi planets or Pegasids, after 51 Peg b) of a given composition will depend on essentially three quantities: their masses, ages, and the amounts of flux they receive from their parent star. The latter are expressed in Table 1 in the form of $T_{\text {eq, }, 0}$, the effective equilibrium temperature calculated for a zero planetary albedo. In terms of these parameters, the sample of known transiting Pegasi planets is already quite rich. And indeed, evolution calculations of individual planets have yielded quite different results. The first one discovered, HD209458b, was shown to be anomalously large (Bodenheimer et al. 2001; Guillot \& Showman 2002). The subsequent six planets appeared relatively "normal", i.e. fitting the standard evolution models within the error bars, with the exception of OGLE-TR-10b, another toolarge planet (Baraffe et al. 2005; Laughlin et al. 2005b). Then, HD149026b was shown to be significantly smaller than expected, requiring the presence of a large amount $\sim 70 M_{\oplus}$ of heavy elements in its interior (Sato et al. 2005). The last addition to that list is that of HD189733b (Bouchy et al. 2005b), 
Table 1. Characteristics of the transiting Pegasi planets discovered so far.

\begin{tabular}{rlrrrrrrr}
\hline \hline$\#$ & Name & Age [Ga $]$ & {$[\mathrm{Fe} / \mathrm{H}]$} & $T_{\text {eff }}[\mathrm{K}]$ & $T_{\text {eq, } 0}[\mathrm{~K}]$ & $M_{\mathrm{p}}\left[M_{\oplus}\right]$ & $R_{\mathrm{p}}[\mathrm{Mm}]$ & Refs. \\
\hline 1 & HD209458 & $4-7$ & $0.02(3)$ & $6117(30)$ & $1487(120)$ & $210(19)$ & $97(5)$ & {$[1,2]$} \\
2 & OGLE-TR-56 & $2-4$ & $0.25(8)$ & $6119(60)$ & $2079(140)$ & $394(73)$ & $88(11)$ & {$[3,4,5]$} \\
3 & OGLE-TR-113 & $0.7-10$ & $0.15(10)$ & $4804(110)$ & $1340(80)$ & $429(70)$ & $77(5)$ & {$[5,6]$} \\
4 & OGLE-TR-132 & $0.5-2$ & $0.37(7)$ & $6210(59)$ & $1980(100)$ & $350(38)$ & $80\left({ }_{-4}^{+9}\right)$ & {$[6,7,8]$} \\
5 & OGLE-TR-111 & $1.1-10$ & $0.19(7)$ & $5044(80)$ & $1033(160)$ & $168(35)$ & $71(4)$ & {$[3,5,9]$} \\
6 & OGLE-TR-10 & $1.1-5$ & $0.28(10)$ & $6075(90)$ & $1578(50)$ & $200(41)$ & $102(7)$ & {$[3,4,5,10]$} \\
& & & & & & & $81(6)$ & {$[11]$} \\
7 & TrES-1 & $2-6$ & $0.06(5)$ & $5226(40)$ & $1157(140)$ & $238(22)$ & $74(+6)$ & {$[5,12]$} \\
8 & HD149026 & $1.2-2.8$ & $0.36(5)$ & $6147(50)$ & $1740(150)$ & $114(10)$ & $52(4)$ & {$[13,14]$} \\
9 & HD189733 & $0.5-10$ & $-0.03(4)$ & $5050(50)$ & $1199(30)$ & $365(13)$ & $90(2)$ & {$[15]$} \\
& & & & & & $82.5(23)$ & {$[16]$} \\
\hline
\end{tabular}

The numbers in parenthesis represent the uncertainties on the corresponding last digits.

References: [1] Winn et al. (2005); [2] Santos et al. (2004); [3] Udalski et al. (2002); [4] Bouchy et al. (2005a); [5] Santos et al. (2006); [6] Bouchy et al. (2004); [7] Moutou et al. (2004); [8] Pont et al. (2006); [9] Pont et al. (2004); [10] Konacki et al. (2005); [11] Holman et al. (2005); [12] Sozzetti et al. (2004); [13] Sato et al. (2005); [14] Charbonneau et al. (2006); [15] Bouchy et al. (2005b); [16] Bakos et al. (2006).

which, like HD209458b orbits a star of near-solar metallicity, and is found to be relatively large.

While a radius that is smaller than expected by theoretical evolution calculations for a solar-composition giant planet can be easily accounted for by the presence of a central dense core, or generally more heavy elements in the interior, large radii point towards missing physics in the models: an additional energy source, or inaccurate physical inputs (equations of state, opacities, atmospheric models). It has been proposed that the anomalous radius of HD209458b could be explained by tidal heating linked to a small forced eccentricity $e \sim 0.03$ (Bodenheimer et al. 2001), but detailed observation indicate that the eccentricity is small, $e=0.014 \pm 0.009$ (Laughlin et al. 2005a), and observations of the secondary eclipse imply that this would further require a chance configuration of the orbit (Deming et al. 2005). Another proposed explanation also involving tidal dissipation of orbital energy is that the planet may be trapped in a Cassini state with a large orbital inclination (Winn \& Holman 2005). Finally, a third possibility that would apply to all Pegasi planets is to invoke a downward transport of kinetic energy and its dissipation by tides (Showman \& Guillot 2002). This last possibility would require the various transiting planets to have different core masses to reproduce the observed radii (Guillot 2005).

The purpose of this article is to test whether these scenarios are possible, and to attempt to constrain the masses of heavy elements present in these planets. We first compare measured radii to the ones obtained by standard evolution models of extrasolar planets. We derive in Sect. 3 masses of heavy elements and compare them to the metallicities of the parent stars. Consequences for our understanding of planet formation are then discussed.

\section{Standard models and the radius anomaly}

We first calculate evolution models for all nine planets based on a simple model assuming a near-solar composition (Guillot 2005). We use a pure hydrogen-helium equation of state (Saumon et al. $1995)$ with a helium mass mixing ratio $Y=0.30$ and standard Rosseland opacities (Allard et al. 2001). The atmospheric boundary condition is calculated for each planet for an incoming stellar flux averaged over the entire planetary surface (Iro et al. 2005). We stress that significant uncertainties on the atmospheric temperature profile arise from incomplete opacity sources, presence or absence of clouds, horizontal inhomogeneities, and advective transport of heat. This implies that atmospheric

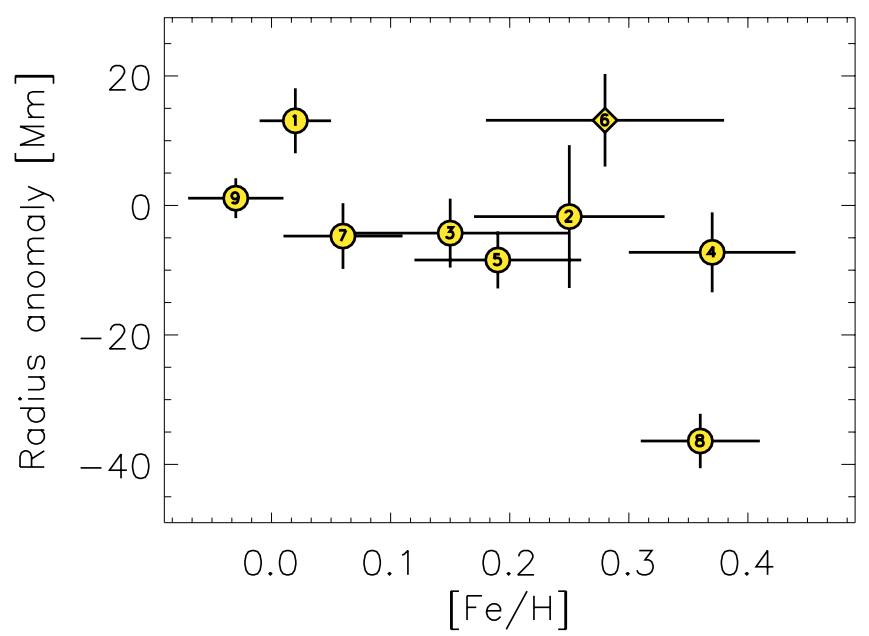

Fig. 1. Radius anomaly (in units of Mm, or $1000 \mathrm{~km}$ ) of the known transiting Pegasi planets as a function of the metallicity of their parent star (see Table 1 for a list of the planets). The radius anomaly is defined as the difference between the measured radius and the one calculated using a simple evolution model for a solar composition gaseous planet.

temperatures in the literature can vary by up to $500 \mathrm{~K}$ (Barman et al. 2001; Sudarsky et al. 2003; Fortney et al. 2005; Iro et al. 2005; Seager et al. 2005). For our purposes however, this will not matter as long as the temperature of the deep atmosphere remains roughly proportional to the equilibrium temperature. For example, in our calculations of atmospheric profiles, the ratio of the temperature at the 10 bar level to the equilibrium temperature is found to be between 1.18 and 1.28 for all the planets in Table 1.

We define the radius anomaly as the difference between the measured radius and the one obtained by our evolution model for the ages inferred in Table 1, and accounting for the fact that the measured radii correspond to $\sim$ mbar levels (Burrows et al. 2003; Fortney et al. 2003; Iro et al. 2005). Figure 1 shows the radius anomaly thus obtained against the metallicity of the parent star. The error bars on the radius anomaly are equivalent $1 \sigma$ error bars calculated on the basis of Gaussian distribution of radii measurements and on a uniform distribution of the age within the limits in Table 1. (Note that for simplicity, in the case of OGLE-TR-132b and Tres-1b, we arbitrarily recentered the radius measurement to have symetrical error bars.) Figure 1 
indicates that the radius anomaly and the metallicity of the parent star may be anticorrelated. We found no such correlation between the radius anomaly and the age, mass, effective temperature of the star, or with the mass of the planet, and equilibrium temperature. We also found no correlation with the total XUV flux received during the planets' lifetime, implying that planetary evaporation probably remained limited in magnitude.

In Fig. 1 and hereafter, two planets deserve a special discussion: OGLE-TR-10b and HD189733b are characterized by two significantly different radius determinations (see Table 1). In the case of OGLE-TR-10b, we adopted the value by Bouchy et al. (2005a), because the value by Holman et al. (2005) remains unconfirmed thus far. A special symbol for OGLE-TR-10b is used to remind us of this uncertainty. For HD189733b, the most recent value (Bakos et al. 2006) was adopted because the photometric analysis appears to be improved over the old value. In both cases, this corresponds to a worst-case situation for the (anti)correlation that we are seeking.

The fact that the radius anomaly decreases with increasing $[\mathrm{Fe} / \mathrm{H}]$ indicates that the source of the correlation is not in the atmosphere because larger amounts of heavy elements would lead to larger atmospheric temperature (Fortney et al. 2005) and therefore larger radii. On the other hand, the magnitude of the anomaly is much too large to be explained by assuming that the planets have the same composition as their stars. On this basis we now attempt to infer the masses of heavy elements present in the planets.

\section{Masses of heavy elements}

At this point, the correlation is just indicative however because the radius anomaly also depends on the planetary mass. In order to ascertain its reality and to constrain the planet composition/star metallicity relationship, we further calculated evolution models for planets with a central rock core and a solarcomposition envelope (see Guillot 2005). The results are shown in Fig. 2, in which we plot as a function of the metal content of the central star, the amount of heavy elements that is required to reproduce the observed planetary radii. In most cases, this amount is positive, but for HD209458b and OGLE-TR-10b, the large measured radii yield by extrapolation unphysical negative values of the mass of heavy elements.

One possible interpretation is that energy dissipation occurs only in HD209458b and OGLE-TR-10b (e.g. via tidal dissipation due to a non-zero inclination of the orbital plane), which could explain the negative core masses derived for these planets. If we retain this explanation, the remaining 7 planets with positive core masses appear to possess amounts of heavy elements that are correlated with the stellar metallicity. The corresponding linear correlation coefficient for these 7 points is then relatively high, $r=0.78$ (Pearson) or $r_{\mathrm{S}}=0.71$ (Spearman), with a corresponding significance level (no-correlation probability) close to $7 \%$. A tough test is however to retry all the points within their error bars and calculate an averaged significance level, which we find to be $\sim 33 \%$.

An alternative explanation that we favor is that some physics is missing in the treatment of the evolution calculation and affects all the planets. One possibility could be that the hydrogen equation of state severely underestimates the density for a given pressure and temperature. It could also be due to a strongly underestimated deep opacity that would then artificially shorten the planetary cooling and contraction. But given that it would require relatively severe modifications to these physical inputs, we will only focus here on a third possibility: that downward

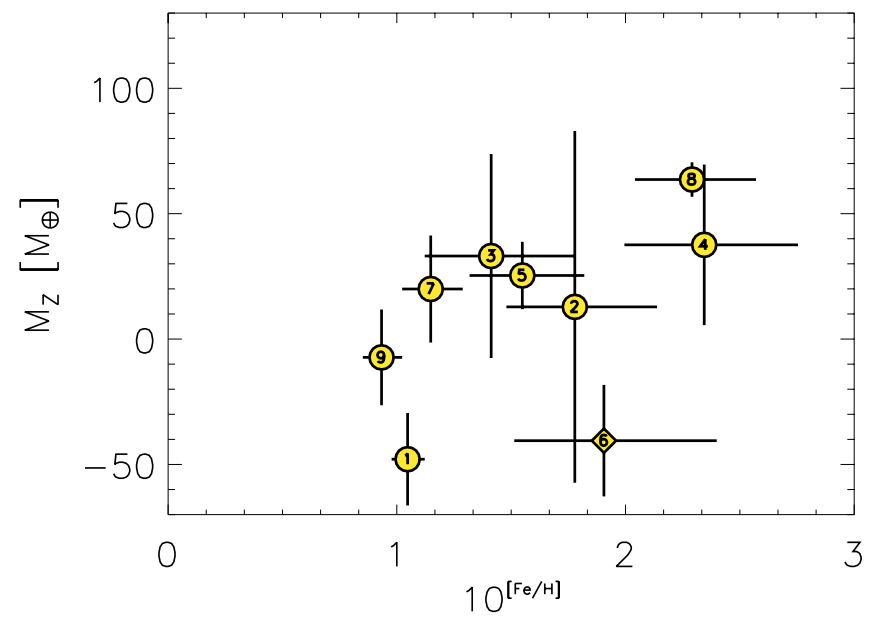

Fig. 2. Mass of heavy elements in the planets as a function of the metal content of the parent star relative to the Sun. The mass of heavy elements required to fit the measured radii is calculated on the basis of standard evolution models. Negative core masses are required in some cases, implying that some significant physical input is missing (see text). Horizontal error bars correspond to the $1 \sigma$ errors on the $[\mathrm{Fe} / \mathrm{H}]$ determination. Vertical error bars are a consequence of the uncertainties on the measured planetary radii and ages.

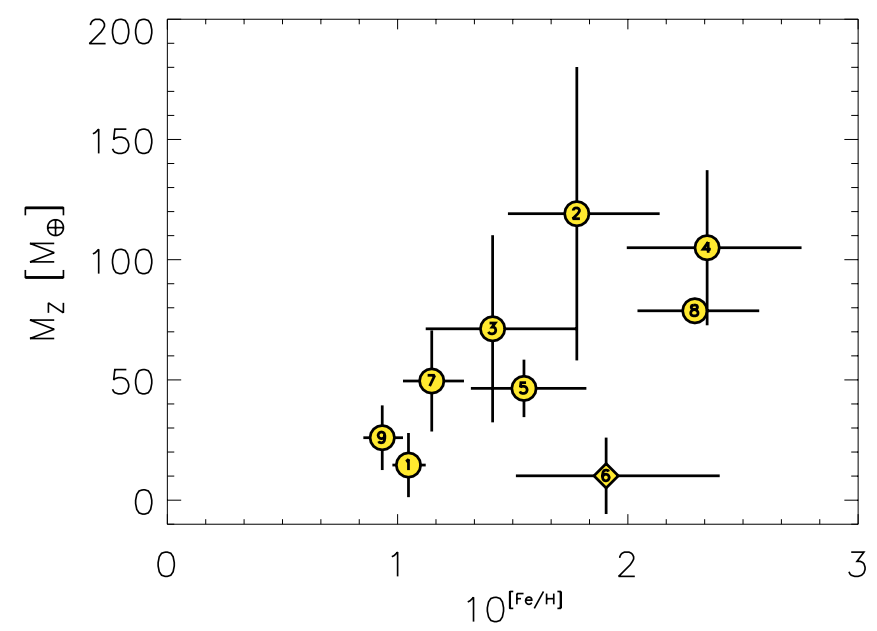

Fig. 3. Same as Fig. 2, but the mass of heavy elements required to fit the measured radii are calculated on the basis of evolution models including an additional heat source slowing the cooling of the planet. This heat source is assumed equal to $0.5 \%$ of the incoming stellar heat flux (Showman \& Guillot 2002).

kinetic energy transport and its dissipation due to tidal effects in the planetary interior effectively leads to an additional energy source that slows the contraction of the planets (Guillot \& Showman 2002; Showman \& Guillot 2002). Quantitatively, we found that adding an additional energy source at the planet center equal to $0.5 \%$ of the incoming stellar luminosity was sufficient to solve the problem of HD209458b and OGLE-TR-10b.

Figure 3 shows the masses of heavy elements that result from these evolution models including this additional energy source. A pleasing feature of these models is that all planets can be explained within the same physical framework, and that the planetstar metallicity correlation appears to be reinforced: including all nine planets, the correlation coefficients are $r=0.58, r_{\mathrm{S}}=0.50$, for a significance level of $17 \%$, which is confirmed by permutation and bootstrap tests. Using Monte-Carlo retrials of the nine points within their error bars, the mean significance level 
increases to $27 \%$. Removing the questionned OGLE-TR-10b point from the sample, the rank correlation coefficient increases to $r_{\mathrm{S}}=0.83$, for a false positive probability of $\sim 1 \%$ and a mean significance level of the Monte-Carlo retrials of $12 \%$. Other calculations (not presented) with a higher fraction of dissipated energy (1\% of the incoming flux), or with an ad-hoc no-helium equation of state also lead qualitatively to the same results.

\section{Conclusions}

We have shown that the nine transiting extrasolar planets discovered so far, including the anomalously large HD209458b and the "big core" planet HD149026b form a relatively homogeneous ensemble, whose compositions can be explained by the same evolution model for all planets. Alternatively, we cannot rule out that the structure of one or several planets was affected by particular physical processes (e.g. a non-zero inclination giving rise to tides, a different orbital evolution etc.).

We found that the masses of heavy elements in the planets appear to be proportional to the metallicities of their parent star. This correlation remains to be tested, being still consistent with a no-correlation hypothesis at the $1 / 3$ level in the least favorable case. If true, it is relatively steep $\left(M_{\mathrm{Z}} \sim 60 M_{\oplus}\left(10^{[\mathrm{Fe} / \mathrm{H}]}-0.8\right)\right)$ and appears to exclude the presence of Pegasi planets around stars with a metallicity smaller than that of the Sun by more than $\sim 0.1 \mathrm{dex}$, in agreement with the lack of Pegasids $(a<$ $0.1 \mathrm{AU}$ ) around metal-poor stars observed by radial velocimetry.

The steepness of the relation, and the large values of $M_{\mathrm{Z}}$ obtained in some cases are surprising and have not been predicted by formation models, either in the framework of the coreinstability model (Ida \& Lin 2004; Alibert et al. 2005) or by models involving direct gravitational instability of the gas (Boss 2003). The core instability model seems however to provide the key ingredients to explain this relation: a faster growth of solid planetary embryos with increased metallicity, the subsequent collection of these during or after the capture of a gaseous envelope, and a more likely migration for planets that are formed quickly. On the other hand, in the framework of gravitational instability models, one needs to assume that the gaseous protoplanets are able to collect dust quickly and that the efficiency of this process is directly linked to the metallicity of the disk. However, we would then expect to find planets around metal-poor stars which is not observed both by transit surveys (although Figs. 2 and 3 imply they would be easier to detect) and by radial velocimetry. It seems therefore unlikely that gravitational instability is responsible for the formation of Pegasi planets.

Another consequence of this work is that the most typical Pegasids, $150 M_{\oplus}$ planets orbiting stars more metal-rich than the Sun, will be smaller than expected. Since the transit depth scales with the square of the radius, this has tough implications for ground-based transit searches. For instance, a $60 \mathrm{Mm}\left(0.86 R_{\mathrm{J}}\right)$ planet would produce a $0.7 \%$ transit in front of a $1 R_{\odot}$ star, instead of $1.2 \%$ for a $80 \mathrm{Mm}\left(1.15 R_{\mathrm{J}}\right)$ planet as typically assumed, enough to sink it below the detection threshold of all present ground-based surveys. And indeed, the planets found around the three most metal-rich stars by photometric transit searches, OGLE-TR-132, 10, and 56 (see Table 1) are also the ones that endure the most irradiation from their parent star: they thus have been prevented from contracting below the threshold level. This may contribute to explain the relative lack of success of photometric transit searches compared to radial velocity surveys.

Clearly, although tantalizing, the results presented in this study are limited by the relatively small number of known transiting planets. In the next few years, the discovery of transiting extrasolar planets, in particular with the space missions COROT (launch 2006) and Kepler (launch 2008), their careful characterization coupled to studies of their parent stars should greatly improve our understanding of planet formation.

Note added in proof: The transiting planet recently discovered by McCullough, P. R., et al. (2006, ApJ, [arXiv: astro-ph/0605414], in press), $\mathrm{XO}-1 \mathrm{~b}$, is very similar in mass, radius and stellar metallicity to HD 209458b and further strengthens the correlation proposed in this paper.

\section{References}

Alibert, Y., Mordasini, C., Benz, W., \& Winisdoerffer, C. 2005, A\&A, 434, 343 Allard, F., Hauschildt, P. H., Alexander, D. R., Tamanai, A., \& Schweitzer, A. 2001, ApJ, 556, 357

Bakos, G. A., Knutson, H., Pont, F., et al. 2006, ArXiv Astrophysics e-prints

Baraffe, I., Chabrier, G., Barman, T. S., et al. 2005, A\&A, 436, L47

Barman, T. S., Hauschildt, P. H., \& Allard, F. 2001, ApJ, 556, 885

Bodenheimer, P., Lin, D. N. C., \& Mardling, R. A. 2001, ApJ, 548, 466

Boss, A. P. 2003, ApJ, 599, 577

Bouchy, F., Pont, F., Santos, N. C., et al. 2004, A\&A, 421, L13

Bouchy, F., Pont, F., Melo, C., et al. 2005a, A\&A, 431, 1105

Bouchy, F., Udry, S., Mayor, M., et al. 2005b, A\&A, 444, L15

Burrows, A., Sudarsky, D., \& Hubbard, W. B. 2003, ApJ, 594, 545

Charbonneau, D., Winn, J. N., Latham, D. W., et al. 2006, ApJ, 636, 445

Deming, D., Seager, S., Richardson, L. J., \& Harrington, J. 2005, Nature, 434, 740

Fortney, J. J., Marley, M. S., Lodders, K., Saumon, D., \& Freedman, R. 2005, ApJ, 627, L69

Fortney, J. J., Sudarsky, D., Hubeny, I., et al. 2003, ApJ, 589, 615

Guillot, T. 2005, Ann. Rev. Earth Planet. Sci., 33, 493

Guillot, T., \& Showman, A. P. 2002, A\&A, 385, 156

Holman, M. J., Winn, J. N., Stanek, K. Z., et al. 2005, ApJ, submitted [arXiv:astro-ph/0506569]

Ida, S., \& Lin, D. N. C. 2004, ApJ, 616, 567

Iro, N., Bézard, B., \& Guillot, T. 2005, A\&A, 436, 719

Konacki, M., Torres, G., Sasselov, D. D., \& Jha, S. 2005, ApJ, 624, 372

Laughlin, G., Marcy, G. W., Vogt, S. S., Fischer, D. A., \& Butler, R. P. 2005a, ApJ, 629, L121

Laughlin, G., Wolf, A., Vanmunster, T., et al. 2005b, ApJ, 621, 1072

Melo, C., Santos, N. C., Israelian, G., et al. 2006, in preparation

Moutou, C., Pont, F., Bouchy, F., \& Mayor, M. 2004, A\&A, 424, L31

Pont, F., Bouchy, F., Queloz, D., et al. 2004, A\&A, 426, L15

Pont, F., Santos, N. C., Melo, C., et al. 2006, in preparation

Santos, N. C., Israelian, G., \& Mayor, M. 2004, A\&A, 415, 1153

Santos, N. C., Pont, F., Melo, C., et al. 2006, A\&A, 450, 825

Sato, B., Fischer, D. A., Henry, G. W., et al. 2005, ApJ, 633, 465

Saumon, D., Chabrier, G., \& van Horn, H. M. 1995, ApJS, 99, 713

Seager, S., Richardson, L. J., Hansen, B. M. S., et al. 2005, ApJ, 632, 1122

Showman, A. P., \& Guillot, T. 2002, A\&A, 385, 166

Sozzetti, A., Yong, D., Torres, G., et al. 2004, ApJ, 616, L167

Sudarsky, D., Burrows, A., \& Hubeny, I. 2003, ApJ, 588, 1121

Udalski, A., Szewczyk, O., Zebrun, K., et al. 2002, Acta Astron., 52, 317

Winn, J. N., \& Holman, M. J. 2005, ApJ, 628, L159

Winn, J. N., Noyes, R. W., Holman, M. J., et al. 2005, ApJ, 631, 1215 